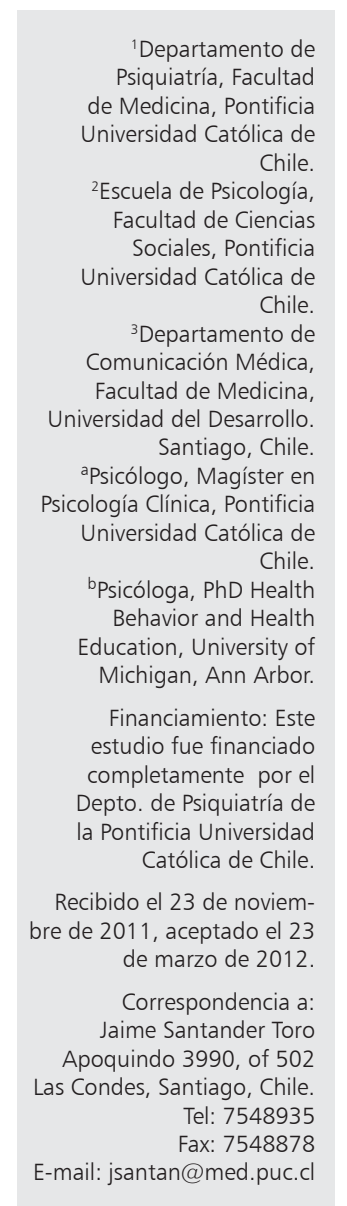

\section{Enseñanza de Psicología Médica en las escuelas de medicina chilenas}

\author{
JAIME SANTANDER T. ${ }^{1}$, JOSÉ PINEDO P. ${ }^{1, a}$, PAULA REPETTO L. ${ }^{2,3, b}$
}

\begin{abstract}
Background: Physicians should be exposed, during their training to basic concepts in psychology. Aim: To describe the current status of the formal teaching of health psychology or medical psychology in Chilean medical schools. Material and Methods: We reviewed the programs of the courses including topics of Medical Psychology, Health Psychology and Behavioral Medicine at 18 medical schools in Chile, using a focused coding method. The contents and the time spent on these courses were considered and analyzed. Results: Eighty three percent of medical schools have a Medical Psychology or related program, 56.3\% are carried out during the first year of medical School teaching and the weekly load has an average of 4 hours. The contents are mixed and predominantly concerning general and developmental psychology, but also address specific issues of Medical Psychology in most cases. Conclusions: There is little clarity about the training issues to be addressed in medical psychology for medical students in Chile. It is necessary to define the minimum content that all medical graduates should learn.
\end{abstract}

(Rev Med Chile 2012; 140: 946-951).

Key words: Behavioral medicine; Education, medical; Psychology, medical.
E xisten diversas razones por las cuales se considera que los médicos deben estar expuestos durante su formación, a conceptos de psicología y, en términos generales a las ciencias de la conducta. Una de las razones más importantes se relaciona con que un número significativo de las causas más importantes de morbilidad y mortalidad en la población mundial ${ }^{1} \mathrm{y}$, en Chile en particular, se relacionan con conductas y, por lo tanto, deben ser consideradas para comprender la situación de la salud de las personas ${ }^{2,3}$. El peso de las conductas de riesgo o cuidado de la salud han alcanzado una importancia crucial en la morbilidad de países con un perfil demográfico como el nuestro, con una alta representación de enfermedades crónicas no trasmisibles y que además tienen un gradiente educacional evidente, todo lo cual aparece claramente representado en la Encuesta Nacional de Salud 2009-2010 . Por lo tanto, incluir en la formación de los estudiantes de medicina, y de otros profesionales del área de la salud, conceptos de psicología que ayuden a la comprensión de los problemas de salud más importantes de la población, puede contribuir a mejorar la atención y cuidado de salud de los pacientes.

En segundo lugar, esta formación es importante debido a que distintos investigadores han mostrado la relevancia de los factores psicológicos en la génesis, evolución y tratamiento de las enfermedades somáticas; así por ejemplo se ha documentado la influencia de los factores psicológicos y sociales en el inicio y evolución de enfermedades metabólicas ${ }^{4,5}$, autoinmunes ${ }^{6}$, gastrointestinales ${ }^{7}$ cardiovasculares $^{8-10}$ y oncológicas $^{11,12}$, entre otras. También se ha demostrado la importancia de considerar los elementos de personalidad del paciente al diseñar estrategias 
que permitan mejorar la adhesión a tratamientos, en especial en las enfermedades crónicas. Cabe mencionar que se estima que sólo entre 43 y 78 $\%$ de los pacientes cumple con las indicaciones en enfermedades crónicas ${ }^{13,14}$.

En tercer lugar, las aplicaciones de teoría psicológica a la comprensión de las conductas de salud y riesgo en pacientes han ayudado al diseño de intervenciones efectivas en este ámbito y, por lo tanto, constituyen un cuerpo de conocimiento que puede contribuir al cuidado y tratamiento de la salud física y mental de las personas ${ }^{15-18}$.

Finalmente, otros autores señalan que la inclusión de contenidos tanto teóricos como prácticos de psicología en la formación en medicina son claves para poder humanizar el trabajo de los médicos y, en general, de los profesionales de la salud. Hoy en día existe consenso entre investigadores, clínicos y formadores de médicos en señalar que el currículo de formación en medicina debe incorporar conocimientos y habilidades de psicología y de las ciencias de la conducta que permitan que en su trabajo, los médicos puedan comprender los problemas de salud que aquejan a las personas de una manera más amplia ${ }^{19}$. De hecho, en un ámbito de acción similar se ha desarrollado la Medicina Psicosomática, también conocida como Psiquiatría de Enlace, la cual incluso ha sido reconocida como una subespecialidad de la Psiquiatría por organizaciones como el American Board of Medical Specialties en $2003^{20}$ y tiene un extenso programa de formación en las áreas ya señaladas y se encuentra incorporada en el funcionamiento cotidiano de las instituciones modernas de salud.

Sin embargo, a nivel de la formación de pregrado las cosas no parecen ser tan claras. Actualmente, en la mayoría de los programas de las escuelas de medicina chilenas se ha incluido en su malla curricular mínima un ramo denominado de Psicología Médica (PM), Psicología de la Salud, de Medicina Conductual, o al menos algunas clases referidas a estos temas dentro de asignaturas del área humanista, que están orientados al desarrollo conocimientos y competencias del ámbito de la psicología que pueden complementar su formación en medicina. Pero, aunque se pueden identificar estos avances, poco o nada está dicho en la literatura nacional acerca de los contenidos que se debe impartir o acerca de cuándo y cómo los médicos llegan ellos a aprender al respecto. En el EUNACOM ST (Examen Único Nacional de
Conocimientos en Medicina según sus iniciales en español) ${ }^{21}$, se definen los contenidos mínimos que los egresados de las escuelas de medicina chilenas deben manejar de las diversas especialidades médicas a las cuales se ven expuestos durante su formación. Sin embargo, este incluye sólo parte de los conocimientos requeridos en ciencias sociales y de la conducta, de manera que tampoco constituye un marco mínimo razonable que permita definir los objetivos y contenidos mínimos comunes a entregar en esta asignatura.

En consideración de las dificultades planteadas y la aparente falta de consenso respecto de las temáticas que deben ser incluidas, nos hemos planteado los siguientes objetivos:

1. Describir la situación actual de la enseñanza formal de la "psicología de la salud" o "psicología médica" en las escuelas de medicina chilenas.

2. Discutir los objetivos y contenidos mínimos de esta disciplina para el currículo de pregrado de las escuelas de medicina chilenas.

\section{Material y Método}

Para efectos del presente estudio, contactamos las autoridades académicas o profesores de las 18 escuelas de medicina que en 2010 ya tenían egresados, solicitándoles los programas del o los ramos de Psicología, Psicología Médica o el más afín a este, que incluyera una o más de las temáticas ya mencionadas. También se les solicitó información relacionada con estos cursos, como la ubicación del ramo en la malla curricular, la cantidad de actividades académicas y los contenidos específicos. Una vez obtenidos los programas de los cursos, agrupamos esta información y la codificamos usando un método de codificación focalizada que permite organizar los datos en función de códigos predefinidos $^{22}$. Los códigos fueron elaborados guiados por las temáticas definidas como relevantes para la formación de los médicos. Este método nos parece más adecuado para lograr los objetivos definidos en el presente estudio.

Luego, la información fue analizada usando estadísticos descriptivos de modo de obtener una visión adecuada de la situación actual en la formación de los médicos en Chile, en esta área del conocimiento. 


\section{Resultados}

\section{Muestra y resultados generales}

Se investigó la enseñanza de la PM, Psicología de la Salud, Medicina Conductual y Ciencias de la Conducta en la totalidad de las escuelas de medicina en Chile que al año 2010 tuvieran egresados. Cumplen con ese requisito 18 escuelas, de las cuales $15(83,3 \%)$ incorporan un curso o parte de un curso con estos contenidos en su malla curricular. En diez de las quince facultades que incorporan en su malla curricular programas con contenidos de PM el nombre de los cursos corresponde a Psicología Médica, y estos son mayoritariamente impartidos durante el primer año de la carrera. $(56,3 \%)$. El desglose detallado de esta información se resume en la Tabla 1.

\section{Carga curricular}

Aunque las distintas escuelas de medicina expresan la carga académica de manera disímil, esto es, en horas cronológicas de realización directa del curso, o en unidades académicas o créditos, equivalentes a la cantidad de horas que un alumno debe invertir en esa asignatura, encontramos que la moda correspondió a tres horas de dedicación a la semana, en tanto la mediana fue de cuatro horas de dedicación a la semana. Sólo en una escuela de medicina la carga fue estimada en 10 horas de dedicación semanal a la asignatura de PM, en tanto en otras dos escuelas en que se estiman 10 o más horas de dedicación semanal se trataba de asignaturas que incluían contenidos de psicología médica pero que tenían un rango de contenidos mucho más diverso ("Psicopatología y Psiquiatría" en un caso, y "Neuropsicopatología" en el otro).

Se debe señalar además, que en 14 de las 15 $(93,3 \%)$ escuelas que tienen un programa de PM, este abarca sólo un semestre.

\section{Contenidos abordados}

De los programas impartidos, sólo pudimos acceder a los de 13 (86,7\%) escuelas de medicina que tienen programas en esta área. Al revisar la información obtenida a partir de los programas revisados podemos ver que las temáticas se pueden dividir en 6 grandes áreas que se resumen en la Tabla 2. En ella observamos que claramente predominan las áreas temáticas relacionadas con Psicología Normal: Psicología del Desarrollo (92,3\% de las facultades), Personalidad (76,9\%) y Psicología General (76,9\%). Los contenidos más directamente relacionados a PM se concentran en dos áreas temáticas: Modelos de salud y enfermedad (en $84,6 \%$ de las facultades que imparten este tipo de cursos) y Relación médico-paciente $(74,6 \%)$, aunque esta última área temática es abordada también en otros cursos.

De manera extraordinaria, una escuela incorpora contenido de medicinas complementarias y otra incluye contenidos de medicina evolucionaria como marco comprensivo adicional al enfoque biopsicosocial.

Tabla 1. Denominación y ubicación en la malla curricular de los cursos que imparten predominantemente contenidos de Psicología médica

\begin{tabular}{|c|c|c|}
\hline \multirow{3}{*}{$\begin{array}{l}\text { Escuelas de Medicina que incorporan Psicología Médica en su malla } \\
\text { curricular }(n=18)\end{array}$} & & n $\quad(\%)$ \\
\hline & Sí & $15(83,3 \%)$ \\
\hline & No & $3(16,7 \%)$ \\
\hline \multirow{4}{*}{$\begin{array}{l}\text { Denominación del curso que incorpora los contenidos de Psicología } \\
\text { Médica }(n=15)\end{array}$} & Nombre del curso & n $\quad(\%)$ \\
\hline & Psicología médica & $10(66,7 \%)$ \\
\hline & Psicopatología y psiquiatría & $2(13,3 \%)$ \\
\hline & Otras denominaciones & $3(20,0 \%)$ \\
\hline \multirow{5}{*}{$\begin{array}{l}\text { Ubicación en la malla curricular }(n=16) \text { (una facultad se divide en dos } \\
\text { períodos) }\end{array}$} & Período Académico & n $\quad(\%)$ \\
\hline & 1 ro o 2 do semestre & $9(56,3 \%)$ \\
\hline & 3ro o 4to semestre & $2(12,5 \%)$ \\
\hline & 5to o 6to semestre & $2(12,5 \%)$ \\
\hline & $7 \mathrm{mo}$ o $8 \mathrm{vo}$ semestre & $3(18,7 \%)$ \\
\hline
\end{tabular}


Tabla 2. Principales áreas y subáreas temáticas abordadas en los programas de Psicología Médica en las Escuelas de Medicina chilenas

\begin{tabular}{|c|c|c|c|}
\hline $\begin{array}{l}\text { Área temática } \\
\text { Contenido }\end{array}$ & n $\quad(\%)$ & $\begin{array}{l}\text { Sub área temática } \\
\text { Contenido }\end{array}$ & n $\quad(\%)$ \\
\hline "Psicología del desarrollo" & $12(92,3)$ & $\begin{array}{l}\text { - Ciclo vital } \\
\text { - Duelo y muerte } \\
\text { - Teoría del apego }\end{array}$ & $\begin{array}{r}11(84,6) \\
6(46,2) \\
2(15,4)\end{array}$ \\
\hline "Modelos de salud enfermedad" & $11(84,6)$ & $\begin{array}{l}\text { - Psicología médica y de la salud } \\
\text { - Psicopatología }\end{array}$ & $\begin{array}{l}9(69,2) \\
3(23,1)\end{array}$ \\
\hline "Personalidad" & $10(76,9)$ & $\begin{array}{l}\text { - Normalidad y anormalidad: teorías, clasificación y } \\
\text { evaluación de la personalidad } \\
\text { - Estructura de personalidad }\end{array}$ & $\begin{array}{l}8(61,5) \\
4(30,8)\end{array}$ \\
\hline "Relación médico paciente" & $10(76,9)$ & $\begin{array}{l}\text { - Habilidades interpersonales y comunicacionales } \\
\text { - Comunicación interpersonal } \\
\text { - Estructura de los encuentros médico pacientes } \\
\text { - El paciente en la relación } \\
\text { - El médico en la relación }\end{array}$ & $\begin{array}{l}8(61,5) \\
6(46,2) \\
5(38,5) \\
4(30,8) \\
4(30,8)\end{array}$ \\
\hline "Psicología general" & $10(76,9)$ & $\begin{array}{l}\text { - Funciones psíquicas } \\
\text { - Dualidad psique soma; relación mente cuerpo } \\
\text { - Aprendizaje } \\
\text { - Introducción a la psicología }\end{array}$ & $\begin{array}{l}7(53,8) \\
4(30,8) \\
3(23,1) \\
3(23,)\end{array}$ \\
\hline "Nuevos paradigmas en salud" & $2(15,4)$ & $\begin{array}{l}\text { - Medicina evolucionaria } \\
\text { - Medicinas complementarias }\end{array}$ & $\begin{array}{ll}1 & (7,7) \\
1 & (7,7)\end{array}$ \\
\hline
\end{tabular}

\section{Discusión}

Es importante señalar que $83,3 \%$ de las escuelas de medicina chilenas incorporan la PM en su plan de estudios, lo que hace pensar que existe conciencia de la importancia de esta disciplina en la formación de los médicos generales. Pese a eso, la heterogeneidad de los programas, así como el bajo peso que estos tienen en los respectivos curriculum académicos pueden llevar a cuestionar esta afirmación. La mayoría de los cursos se imparte en primer año y la manera cómo se presentan los temas no parecen estar conectados de manera directa con la práctica médica, aspecto que se recomienda cuando se incorporan estas temáticas en la formación de los futuros médicos ${ }^{23}$. Por otro lado, la mayoría de las temáticas abordadas se relacionan con psicología normal y en pocos programas se abordan aquellas temáticas consideradas dentro de lo que es la psicología médica y de la salud. Una de las limitaciones de este trabajo es que no se indaga en forma profunda acerca de la eventual entrega de conocimientos de PM en cursos superiores, en talleres, seminarios, tutorías o incluso en otros cursos que aborden esta temática. Esto dificulta sacar conclusiones respecto a la enseñanza total de PM en los programas de medicina del país así como acerca de la importancia y profundidad que se le pueda estar dando. Aún así nos parece que al menos los resultados expuestos son un indicador general del abordaje y sistematización del tema en las escuelas de medicina chilenas.

La enseñanza de la medicina en el pregrado de las universidades chilenas comprende siete años, que en general se dividen en tres grandes bloques, los dos primeros años dedicados a los ramos básicos, luegos tres años de ramos clínicos y por último, los dos de transferencia de conocimientos a la práctica clínica, el internado. En esta estructura, no es de extrañar que si los cursos de PM han sido ubicados curricularmente, principalmente en el espacio destinado a los ramos básicos $(68,8 \%)$, predominen en los contenidos justamente aquellos que son fundantes de esa disciplina, esto es, los relacionados con psicología normal o psicología general. Los contenidos propiamente asociados a PM o psicología de la salud, aunque están presentes en la mayor parte de los programas de estudio, enfrentan dos limitaciones relevantes: una relativa a que comparten con otras asignaturas de 
mucho mayor carga académica un espacio bastante restringido en la malla curricular, lo que es evidenciado por la baja carga académica otorgada a estos programas, que se concentran en un solo período semestral; y que por tratarse de cursos dirigidos a los alumnos de los primeros años de la carrera, se enfrentan a un auditorio que tiene poca noción acerca del rol que los factores psicológicos tienen en el desarrollo de la clínica médica, noción que se va desarrollando progresivamente con el contacto cada vez más intenso con los pacientes en los años venideros. Estas son limitaciones que deben ser abordadas al definir los objetivos para la formación de los estudiantes de medicina e implementar futuras modificaciones curriculares. Trabajar estos aspectos con estudiantes en cursos superiores, y en variadas etapas de la formación, se hace más necesario si consideramos la evidencia que sugiere que los estudiantes de medicina podrían ir disminuyendo su capacidad empática a lo largo de los años de formación ${ }^{24-26}$.

Además nos encontramos con una gran heterogeneidad en las materias abordadas entre las diferentes escuelas de medicina, con temas que incluso se salen de los márgenes de las disciplinas ya señaladas. En este sentido se hace necesario definir un marco para los cursos de PM. En 2004, un Comité de Expertos ${ }^{19}$ definió las áreas de las ciencias sociales y de la conducta que los estudiantes de medicina debieran conocer: i) la interacción mente y cuerpo en los procesos de salud enfermedad; ii) la comprensión de las conductas del paciente; iii) rol del médico y sus conductas en el cuidado de salud; iv) relación médico paciente; v) sensibilidad social y cultural y; vi) economía de la salud. Nos parece que las primeras cuatro debieran estar contenidas en la asignatura de PM de cualquier escuela de medicina del país, lo cual coincide con la definición de PM o Psicología de la Salud: es una disciplina que se dedica "al entendimiento de las influencias psicológicas en la salud de las personas, por qué se enferman y cómo responden cuando se enferman" y "se preocupa de todos los aspectos de la salud y enfermedad a lo largo del ciclo vital" 27 . Por otra parte, es importante no perder de vista que existe también una importante tradición europea en el desarrollo de la PM o Psicología de la Salud que se inicia en la primera mitad del siglo XX y se plasma en importantes textos, como los presentados por E. Kretshmer en Alemania ${ }^{28}$ y J.J. López-Ibor ${ }^{29}$, este último gran impulsor de la disciplina en España. Estos autores aportan una visión más antropológica centrada en la vivencia de la enfermedad desde el individuo. Debemos considerar que el conjunto de estas orientaciones debiera servir de insumo para la necesaria discusión que debemos realizar para definir los conocimientos mínimos de PM a incluir en la formación de pregrado de médicos.

Creemos que para favorecer la transferencia exitosa de conocimientos a los alumnos sería de gran utilidad que el EUNACOM ST ${ }^{21}$ o alguna instancia similar evalúen estos conocimientos de manera sistemática, ya que son fundamentales para la práctica clínica exitosa. Ello tendría la ventaja adicional de proveer un marco teórico mínimo a considerar en los respectivos currículos. En segundo lugar, pensamos que debe existir un plan de desarrollo de módulos a lo largo de la carrera, en especial en los bloques clínicos y en el internado, con seminarios o tutorías profundicen en estos temas, en particular los relacionados con la relación médico paciente y con la conducta del paciente.

\section{Comentario final}

Según lo revisado, este es el primer estudio que revisa la enseñanza de la PM en Chile y Sudamérica a los estudiantes de pregrado de Medicina, lo que constituye un aporte inicial a la discusión en torno a los elementos que deben incluirse en el plan de estudios. En este trabajo ha quedado en evidencia que pese a que la gran mayoría de las escuelas de medicina incorporan asignaturas de PM, se trata de cursos breves, de baja carga curricular y ubicados en los primeros semestres de la carrera, orientándose los contenidos en forma principal a aspectos de psicología general y en menor manera a la PM propiamente tal. En términos generales se trata de cursos de fundamentos de Psicología, asociados de mayor o menor manera a contenidos de PM. La ubicación de esta asignatura precozmente en la malla dificultad la transferencia de conocimientos al área clínica, que es donde estos son mayormente requeridos, y por ende, se requiere además de definir los contenidos mínimos que todo egresado de pregrado de medicina debiera conocer, establecer programas de exposición periódica a lo largo de la carrera a los tópicos en discusión. 


\section{Referencias}

1. World Health Organization. The Global Burden of Disease: 2004 Update. Geneve: WHO Press; 2008.

2. McGinnis JM, Foege WH. Actual causes of death in the United States. JAMA 1993; 270 (18): 2207-12.

3. Ministerio de Salud. Encuesta Nacional de Salud: Chile 2009-2010. http:/www.minsal.gob.cl/portal.docs/page/ minsalcl/g_home/submenu_portada_2011/ens2010.pdf (consultado 13 de octubre de 2011).

4. Chida Y, Hamer M. An association of adverse psychosocial factors with diabetes mellitus: a meta-analytic review of longitudinal cohort studies. Diabetologia 2008; 51: 2168-78.

5. Quintana A, Merino JM, Merino P, Cea JC. Role of psychosocial variables in the metabolic control of type 2 diabetics. Rev Med Chile 2008; 136: 1007-14.

6. Kiecolt-Glaser J, McGuire L, Robles T, Glaser R. Psychoneuroimmunology and Psychosomatic Medicine: Back to the Future. Psychosom Medicine 2002; 64: 15-28.

7. Van Oudenhove L, Vanderberghe J, Demyttenaere K, Tack J. Psychosocial Factors, Psychiatric Illness and Functional Gastrointestinal Disorders: A Historical Perspective. Digestion 2010; 82: 201-10.

8. Orth-Gomér K. Psychosocial and Behavioral Aspects of Cardiovascular Disease Prevention in Men and Women. Curr Opin Psychiatry 2007; 20: 147-51.

9. Everson-Rose S, Lewis T. Psychosocial Factors and Cardiovascular Diseases. Annu Rev Public Health 2005; 26: 469-500.

10. Calderón J, Gabrielli L, González M, Villarroel L, Corbalán R. Depressive symptoms among patients with acute myocardial infarction. Relationship with revascularization therapy. Rev Med Chile 2010; 138 (6): 701-6.

11. Lillberg K, Verkasalo P, Kaprio J, Teppo L, Helenius H, Markku Koskenvuo M. Stressful Life Events and Risk of Breast Cancer in 10,808 Women: A Cohort Study. Am J Epidemiol 2003; 157: 415-23.

12. Moreno-Smith M, Lutgendorf S, Sood A. Impact of stress on cancer metastasis. Future Oncol 2010; 6 (12): 1863-81.

13. Osterberg L, Blaschke T. Adherence to Medication. N Engl J Med 2005; 353: 487-97.

14. Ortiz M, Ortiz E. Health psychology: A key to understand therapeutic adherence. Rev Med Chile 2007; 135: 647-52.

15. Sullivan MJL, Adams H. Psychosocial treatment techniques to augment the impact of physiotherapy interventions for low back pain. Physiother Can 2010; 62: 180-9.

16. Turner J, Kelly B, Clarke D, Yates P, Aranda S, Jolley D, et al. A randomised trial of a psychosocial intervention for cancer patients integrated into routine care: the PROMPT study (promoting optimal outcomes in mood through tailored psychosocial therapies). BMC Cancer 2011; 11: 48-56.

17. Horodynski M, Baker S, Coleman G, Auld G, Lindau J. The Healthy Toddlers Trial Protocol: An Intervention to Reduce Risk Factors for Childhood Obesity in Economically and Educationally Disadvantaged Populations. BMC Public Health 2011, 11: 581-7.

18. Tresco K, Lefler E, Power T. Psychosocial Interventions to Improve the School Performance of Students with Attention-Deficit/Hyperactivity DisorderMind Brain. 2010; 1 (2): 69-74.

19. Institute of Medicine of the National Academies. The Behavioral and Social Sciences in Medical School Curricula. En: Cuff P, Vanselow N, editores. Improving Medical Education: Enhancing the Behavioral and Social Sciences in Medical School Curricula. Washington, USA. National Academies Press; 2004, p. 52-86.

20. Gitlin D, Levenson J, Lyketsos C. Psychosomatic Medicine: A New Psychiatric Subspecialty. Acad Psychiatry 2004; 28: 4-11.

21. EUNACOM. Perfil de Conocimientos EUNACOM, versión 2, 2010. http://www.eunacom.cl/contenidos/ PerfilNew.pdf (consultado 15 de octubre de 2011).

22. Raddon R, Raby R. Sharpe E. The Challenges of Teaching Qualitative Coding: Can a Learning Object Help?. IJTLHE 2009; 21: 336-50.

23. Peters S, Livia L. Relevant behavioural and social science for medical undergraduates: a comparison of specialist and non-specialist educators. Med Education 2006,: 40; 1020-6.

24. Hojat M, Vergare MJ, Maxwell K, Brainard G, Herrine SK, Isenberg GA, et al. The devil is in the third year: A longitudinal study of erosion of empathy in medical school. Acad Med 2009; 84: 1182-91.

25. Newton BW, Barber L, Clardy J, Cleveland E, O'Sullivan P. Is there hardening of the heart during medical school? Acad Med 2008; 83: 244-9.

26. Neumann M, Edelhäuser F, Tauschel D, Fischer MR, Wirtz M, Woopen C, et al. Empathy decline and its reasons: a systematic review of studies with medical students and residents. Acad Med 2011; 86 (8): 996-1009.

27. Taylor S. ¿Qué es la Psicología de la Salud?. En: Taylor S. Psicología de la Salud. 6th. México: McGraw-Hill Interamericana; 2007: 2-14.

28. Kretschmer E. Medizinische Psychologie. Leipzig, Thieme. 1922

29. López-Ibor JJ. Lecciones de Psicología Médica. Madrid, Paz Montalvo. 1957. 